\title{
Liquid Cell Transmission Electron Microscopy Observation of Lithium Metal Growth/Dissolution: Root Growth, Dead Lithium and Lithium Flotsams
}

\author{
Akihiro Kushima ${ }^{1}$, Kang Pyo So ${ }^{1}$, Cong $\mathrm{Su}^{1}$, Peng Bai ${ }^{2}$, Nariaki Kuriyama ${ }^{3}$, Takanori \\ Maebashi $^{3}$, Yoshiya Fujiwara ${ }^{3}$, Martin Z. Bazant ${ }^{2,4,5^{*}}$, and $\mathrm{Ju} \mathrm{Li}^{{ }^{*}}$ \\ ${ }^{1}$ Department of Nuclear Science and Engineering and Department of Materials Science and \\ Engineering, Massachusetts Institute of Technology, Cambridge, MA 02139, USA \\ ${ }^{2}$ Department of Chemical Engineering, Massachusetts Institute of Technology, Cambridge, MA \\ 02139, USA \\ ${ }^{3}$ Honda R\&D Co., Ltd. Wako-shi, Saitama 351-0193, Japan \\ ${ }^{4}$ Department of Mathematics, Massachusetts Institute of Technology, Cambridge, MA 02139, \\ USA \\ ${ }^{5}$ Department of Materials Science and Engineering and SUNCAT Center, Stanford University, \\ Stanford, CA 94305
}

Keywords: in-situ TEM, liquid cell, lithium dendrite, mossy lithium, electrodeposition

\begin{abstract}
We present in situ environmental transmission electron microscopy (ETEM) observation of metallic lithium nucleation, growth and shrinkage in a liquid confining cell, where protrusions are seen to grow from their roots or tips, depending on the overpotential. The rate of solidelectrolyte interface (SEI) formation affects root vs. tip growth mode, with the former akin to intermittent volcanic eruptions, giving kinked segments of nearly constant diameter. Upon delithiation, root-grown whiskers are highly unstable, because the segmental shrinkage rate depends on $\mathrm{Li}^{+}$transport across SEI, which is the greatest around the latest grown segment with the thinnest SEI, and therefore the near-root segment often dissolves first and the rest of the dendrite then loses electrical contact. These electrically isolated dead lithium branches are also easily swept away into the electrolyte to become "nano-lithium flotsam" because the hollowedout SEI tube is very brittle. Our observations are consistent with SEI-obstructed growth by two competing mechanisms; tip growth of dense Eden-like clusters and root growth of whiskers, resulting from the voltage-dependent competition between lithium electrodeposition and SEI formation reactions. Similar phenomena could occur whenever chemical deposition/dissolution competes with irreversible side reactions that form a passivating layer on the evolving surface.
\end{abstract}

Authors to whom all correspond should be addressed: 1iju@mit.edu and bazant@mit.edu 
The lithium metal anode has an extremely high theoretical capacity $(3800 \mathrm{Ah} / \mathrm{kg})$ and low negative electrochemical potential ( $-3.04 \mathrm{~V}$ vs. standard hydrogen electrode). It has been extensively studied ${ }^{1-4}$ since the lithium metal battery was first proposed in 1970 s. $^{5}$ Despite its promising high energy density, a rechargeable lithium metal battery has not yet been commercialized successfully. ${ }^{6}$ In contrast, the Li-ion battery with a graphite insertion anode is dominating markets ranging from portable electronics to electric vehicles and grid-scale energy storage. $^{2,3}$

A generic keyword explaining this history is "dendrite", although we will advocate for a more nuanced use of this term. ${ }^{7}$ An initially smooth Li metal anode is prone to surface morphological instabilities in electrochemical cycling, forming extended protrusions that can cause not only dramatic loss of reversible capacity, but also penetration of the separator, short-circuiting, and in the worst cases heating and catastrophic burning. ${ }^{8}$ Numerous researches have been conducted in the last 40 years to understand the mechanisms and morphologies of Li metal growth, both experimentally ${ }^{9-15}$ and theoretically, ${ }^{6,16-19}$ and methods to suppress the dendrite formation have also been proposed. ${ }^{2,20-25}$ Generally, the morphology of the electrochemically deposited solid is affected by the current density and overpotential, ${ }^{26-28}$ and various qualitative theories have been developed to explain the growth mechanism. ${ }^{6}$ Dendrites in solidification and phase transformation theory generally result from long-range liquid-matrix diffusion limitation, which causes thermal or chemical transport-induced instability of a smooth interface and leads to tip growth. ${ }^{29}$ Dendrites in copper and zinc electrodeposition clearly result from diffusion-limited tip growth, ${ }^{30-34}$ so until recently, the "dendrites" that form at the Li metal anode were also believed to grow from the tips, despite occurring well below the long-range diffusion-limited current of the electrolyte. ${ }^{35-37}$ Suprisingly, it was demonstrated by Dollé et al. through in-situ scanning electron microscopy (SEM) experiments using a polymer electrolyte that dendrites could also grow from the root. ${ }^{38}$ Similar root growth was observed by in-situ optical microscopy (OM) experiments using liquid electrolyte. ${ }^{13,14}$ Although different mechanisms have been suggested to explain the root growth, ${ }^{13,19}$ experimental investigation of the early stage of the nucleation and growth requires direct nanoscale observations. Here we reveal the essential roles of the liquid electrolyte decomposition reaction and SEI formation in the growth mode selection. In contrast to the liquid-state diffusion-limited growth of $\mathrm{Cu}$ and $\mathrm{Zn}$ dendrites, we show that, for typical operating currents, the growth and dissolution of Li "whiskers" and "mossy" deposits are reaction limited and modulated by short-range solid-state diffusion through the passivating SEI layer that is $<10 \mathrm{~nm}$ thin.

At the voltages we are considering, both $\mathrm{Li}^{+}$and the liquid electrolyte solvents are electrochemically unstable: they compete for electrons and co-deposit their reducing products ( $\mathrm{Li}$ atoms and SEI constituents) wherever the electron sources might be. If the competition favors the latter, the SEI constituents might percolate and cover up the entire electron-donating surface (like CO poisoning of catalyst surface), which has two key consequences: (a) $\mathrm{The}^{+} i^{+}$then has to diffuse across the SEI to be reduced, so Li atoms will deposit beneath the SEI, and (b) the SEI 
covering has mechanical strength, which will constrain such growth beneath, causing tensile stress in the SEI layer and compressive stress in the Li-metal beneath it. This stress balance will be broken when the SEI fractures at some location, forming a "fumarole" (taking a volcano analogy), while the compressive stress in the Li metal will drive an "atomic fountain", a $\mathrm{Li}$ whisker that shoots out of the fumarole by Nabarro-Herring or Coble creep (root growth), similar to how Sn whiskers form inadvertently in the microelectronics industry. If, on the other hand, the competition favors the former, then they will still be SEI patches here and there, but they do not connect or cannot seal up the growth front and $\mathrm{Li}$ atoms will grow around them, forming a mixture. In either case, because the liquid electrolyte solvents are electrochemically unstable and co-deposit with Li metal, their short-range interactions have to be carefully considered.

In-situ transmission electron microscopy (TEM) allows observations of electrochemical reactions at nanoscale in real time, ${ }^{39,40}$ originally with ionic liquid or solid electrolytes because of the high vacuum inside TEM. By sealing the volatile liquid electrolyte with two thin membrane windows that are electron-beam transparent, ${ }^{41-43}$ in-situ TEM observations of electrochemical reactions at common solid-liquid interface have been achieved ${ }^{44-46}$, herein with our custom-built lab-on-a-chip mounted on standard TEM holder. ${ }^{47}$ These methods have been applied to observe the formation/growth of the dendrites successfully. ${ }^{43,48}$ However, only the tip growth mode was reported with ETEM. Understanding both the tip and the root growth modes is essential for achieving the following three levels of engineering goals for Li-metal anode, whose fulfillment is necessary for industry adoption: (A) total suppression of short circuiting across the separator, (B) improvement of Coulombic efficiency (reversibility of Li metal deposition) to $99.9 \%$ and above, and $(\mathrm{C})$ reducing the speed of burning, upon breakage of battery cell and exposure to oxygen, by reducing the lithium surface area to volume ratio. Here we present in-situ ETEM observation of the root growth/shrinkage behavior of the lithium dendrite and fine-scale analysis of the process and its consequences, in different overpotential regimes. In addition to short-range solid-state interactions we have mentioned above, we can also observe change of chemistry in the liquid state and nanoscale flow fields around the evolving metal surface.

In order to conduct the in-situ ETEM experiment, we developed a liquid environmental electrochemical cell (LEEC) to prevent the evaporation of the volatile liquid electrolyte during TEM observations. ${ }^{47}$ The device is schematically illustrated in Fig. 1(a). It consists of two silicon chips with $70 \mathrm{~nm}$ thick silicon nitride membrane windows. On one chip the gold electrodes were patterned on the window for observing the electrochemical redox reactions, and spacers were deposited to secure vertical gap space for the liquid electrolyte. On the opposite chip, a $\mathrm{LiCoO}_{2}$ (LCO) film was deposited on an aluminum film as a lithium ion source. The detail of the device is explained in Supplementary Information. The assembly process is described in Fig. 1(b). The two chips were stacked together and the two of the perimeter edges were sealed with epoxy glue. Liquid electrolyte was injected into the device by contacting the droplet to one of the opening (capillary action). Finally, the openings were sealed with epoxy glue. It is difficult to precisely evaluate the amount of electrolyte injected. However, it can be estimated to be in the order of 
nanoliter based on the geometry of the cell (see Supplementary Information). The liquid injection and the final sealing were conducted in a glove box filled with argon gas. The electrolyte used in this work was a solution of $1 \mathrm{M}$ lithium bis-trifluoromethanesulfonimide (LiTFSI) dissolved in dimethoxy sulfoxide (DMSO), one of the promising electrolytes for Li-air batteries ${ }^{49}$ which use lithium metal as the anode. Figure 1(c) shows the cross section of the device near the aligned viewing windows. Most of the electrode surface was insulated with silicon nitride to allow reaction only on the uninsulated gold electrodic region at the viewing window. We mounted the device on a Nanofactory scanning tunneling microscopy (STM) - TEM holder to apply bias voltage during TEM observation. A low dose rate of $1.3 \mathrm{e}^{-} / \AA^{2} \mathrm{~s}$ was used to minimize the beam damage to the electrolyte, and the images were captured in the TEM bright-field mode.

Figure 2(a) shows sequential TEM images of the electrodeposition process on gold biased at $4.5 \mathrm{~V}$ vs. the LCO counter electrode, which drives lithium metal deposition and non-uniform protrusions (see also Supplementary Movie S1). Because the typical plateau charging potential of $\mathrm{LCO}$ starts at $\sim 3.9 \mathrm{~V} \mathrm{vs} \mathrm{Li}^{+} / \mathrm{Li}$, the total local over-potential is around $3.9-4.5 \approx-0.6 \mathrm{~V}$ vs $\mathrm{Li}^{+} / \mathrm{Li}$, much of which is likely transferred to the surface by the highly ion-conductive liquid electrolyte (see below), so the driving force for lithium metal deposition is quite high. Also, the liquid electrolyte is very unstable at this potential ${ }^{8}$ and will decompose by reduction to form SEI constituents on any newly exposed electron-conductive surface. We observed that small spherical lithium domains first appear on the surface of the gold electrode and then start to evolve into long whiskers that grow like hair from their roots at the electrode interface, in contrast to the tip growth of conventional dendrites in solidification and copper electrodeposition. The electric current during the deposition is shown in Fig. 2(b). Although there is a high initial current, it remains almost constant $\sim 20 \mathrm{nA}$. Based on the electrode geometry (see Supplementary Information), this corresponds to the current density of $\sim 2.8 \mathrm{~mA} / \mathrm{cm}^{2}$. Figure 2 (c) is the magnified view of the growth process of the single lithium "hair" or "whisker" indicated by the arrowhead in Fig. 2(a). The shape of the tip as well as the width of the whisker remain the same throughout the growth process, which is a clear sign of root growth. The whisker growth direction also intermittently changed by forming kinks, consistent with the ex-situ SEM observation of the lithium whiskers formed by the root growth mechanism. ${ }^{38}$

The whiskers started to dissolve and shrink after the bias potential was switched to $1.0 \mathrm{~V}$ vs. LCO. Figure 3(a) shows the sequential TEM images during the dissolution (see also Supplementary Movie S2). The whiskers rapidly dissolved in the first 15 seconds, and their morphology did not change henceforth. Some of the pieces apparently become electrically disconnected and isolated from the electrode (dead volume). Thereafter, electro-osmotic flows of the electrolyte swept away these isolated remnants, leaving only a few attached to the electrode, as shown in the area marked by the dashed line in the figure. The change in the electric current during dissolution is shown in Fig. 3(b). The current decreased rapidly and became nearly zero after $20 \mathrm{~s}$. This together with the in-situ TEM observation indicates that the dissolution of the lithium whiskers created electrically isolated "dead lithium", causing irreversible capacity loss in 
the deposition/dissolution cycling process. Some fragments were weakly attached to the electrode and easily swept away into the electrolyte, while others remained on the electrode but were electrically inactive. Since by then no electric current was observed as the lithium whiskers stopped shrinking, the pieces of the lithium left on the electrode were likely connected only by the hollowed-out SEI shells, which are electrically insulating. From the current vs. time plots shown in Fig. 2(b) and 3(b), we estimated the Coulombic efficiency to be $\sim 20 \%$ with the discharge and the charge capacity of $7.7 \mu \mathrm{C}$ and $1.6 \mu \mathrm{C}$, respectively.

Figure 4(a) shows the nucleation and growth of another single lithium whisker in a higher magnification TEM observation (see also Supplementary Movie S3), at -6.0 V vs LCO. Sequential high-resolution TEM images allow us to perform detailed analysis of the reaction kinetics. The initially formed whisker $\Lambda$ was separated into two segments $\Lambda 1$ and $\Lambda 2$, with $\Lambda 2$ pushing $\Lambda 1$ along the direction indicated by the arrow (see schematics below the TEM images). Figure 4(b) shows the change in the length and the width of the whisker $\Lambda(\Lambda \rightarrow \Lambda 1, \Lambda 2$ after the kink formation) during electrodeposition. The whisker growth process can be separated into four distinct stages:

STAGE I: A spherical lithium nucleus emerged at the surface and grew with its diameter proportional to the square root of the time, indicating diffusion limitation, which we associate below with short-range solid-state transport through the SEI layer, rather than long-range liquidstate diffusion. The SEI layer passivates the surface and gradually reduces the lithium deposition rate, since $\mathrm{Li}^{+}$ions need to diffuse across the thickening SEI film that covers the surface.

STAGE II: The lithium whisker started to grow from its root pushing the initially formed sphere away from the electrode. Here a rapid increase in the length of the whisker $\Lambda$ was observed, while its width remained almost constant. A dramatic upturn in lithium deposition rate between stage I and II indicates some kind of abrupt transition, akin to intermittent stick-slip dynamics in friction.

STAGE III: The growth rate decreased significantly, which can be attributed to the formation/thickening of the SEI covering layer on the newly formed portion of the whisker.

STAGE IV: A kink was formed on the whisker separating it into two segments $\Lambda 1$ and $\Lambda 2$. Newly formed segment $\Lambda 2$ grew from the root and pushed the preexisting portion of the whisker in the direction different from the growth direction of $\Lambda$ (a kink formation). While $\Lambda 2$ increased its length and the width, $\Lambda 1$ length remained unchanged. Note that the growth rate of the $\Lambda 2$ was close to that of $\Lambda$ and the width of the $\Lambda 2$ was saturated to the same value with $\Lambda 1$. Such intermittent kinked growth then repeats quasi-randomly.

A quantitative analysis of the growth mechanisms was performed based on the TEM movies. In Stage I, a small spherical lithium seed was formed at the location marked by the open arrowhead in the figure. The sphere grew larger during the deposition, but the growth rate gradually 
decreased. The size of the sphere grew roughly as the square root of time, $h(t)=h_{1}\left(t / \tau_{1}\right)^{1 / 2}$, where $h_{1}=300 \mathrm{~nm}$ and $\tau_{1}=25 \mathrm{~s}$. The observed size can be converted to a decaying, uniform current density, $I(t)=I_{1}\left(\tau_{1} / t\right)^{1 / 2}$, where $I_{1}=c_{\mathrm{Li}} F h_{1} / 2 \tau_{1}=4.8 \mathrm{~mA} / \mathrm{cm}^{2}$, using Faraday's constant, $F=96,485 \mathrm{~A} \mathrm{~s} / \mathrm{mol}$, and the lithium metal concentration, $c_{\mathrm{Li}}=0.534 \mathrm{~g} / \mathrm{cm}^{3} / 6.491 \mathrm{~g} / \mathrm{mol}=82$ M.

Square-root scaling with time strongly suggests diffusion limitation, but does not reveal the rate-limiting transport process. All existing models for the growth of "dendrites" assume liquid electrolyte diffusion limitation, but this is clearly impossible at the nanoscale. The steady limiting current density on the whisker nucleus, modeled as a hemispherical ultramicroelectrode, ${ }^{50} I_{\text {lim }}=2 F c_{0} D_{\text {app }} / h_{1}=19 \mathrm{~A} / \mathrm{cm}^{2}$, is 5,000 times larger the observed current density, $I_{1}\left(c_{0}=1 \mathrm{M}, D_{\text {app }}=3 \times 10^{-6} \mathrm{~cm}^{2} / \mathrm{s}^{51}\right)$. Moreover, the relaxation time for electrolyte diffusion, $\tau_{\text {lim }}=h_{1}^{2} / D_{\text {app }}=0.3 \mathrm{~ms}$, is 10,000 times smaller than the growth time $\tau_{1}$. On the other hand, the measured current $I_{1}$ at the end of spherical growth is only somewhat larger than reported exchange currents for electron-transfer reactions at lithium metal films, ${ }^{52} I_{0}=0.1-2.0$ $\mathrm{mA} / \mathrm{cm}^{2}$. Overall, we conclude the lithium growth processes observed in our experiments are all macroscopically reaction-limited at the electrode/electrolyte interface, although the local reaction rate can be microscopically diffusion-limited by transport processes within the solidstate interface.

As noted above, the slowing down of the initial surface growth rate is likely related to rapid SEI formation, which competes with lithium electrodeposition and hinders $\mathrm{Li}^{+}$transport. It is well established that capacity fade in Li-ion batteries exhibits square-root-time scaling due to ratelimiting solvent diffusion across the SEI layer on porous carbon, ${ }^{37,53-55}$ but this process (by itself) is too slow to play a role in the early stages of lithium metal growth. Using the fitted solvent diffusivity in SEI on carbon in similar electrolytes, $D_{\text {sol }}^{\text {SEI }} \sim 3 \times 10^{-17} \mathrm{~cm}^{2} / \mathrm{s}{ }^{54,55}$ (or $\sim 10^{-19} \mathrm{~cm}^{2} / \mathrm{s}$ ${ }^{53}$ ), the solvent penetration depth in our experiments would be at the single-molecule scale, $\sqrt{2 D_{\text {sol }}^{\mathrm{SEI}} \tau_{1}}=0.4 \mathrm{~nm}$ (or $0.02 \mathrm{~nm}$ ). Instead, we propose that $\mathrm{Li}^{+}$diffusion through the growing SEI (full coverage) limits the local reaction rate and leads to square-root-time scaling at early times.

To test this hypothesis quantitatively, we formulate a simple model for the coupled rates of electrodeposition and SEI formation. The Li metal surface growth is limited by $\mathrm{Li}^{+}$diffusion through the SEI film of thickness, $s(t)$, whose growth is limited by solvent diffusion through the same SEI film,

$$
c_{\mathrm{Li}} \frac{d h}{d t}=\frac{D_{\mathrm{Li}^{+}}^{\mathrm{SEI}} c_{0}}{s}, \quad c_{\mathrm{SEI}} \frac{d s}{d t}=\frac{D_{\mathrm{sol}}^{\mathrm{SEI}} c_{\mathrm{sol}}}{s} \Rightarrow h(t)=\frac{D_{\mathrm{Li}^{+}}^{\mathrm{SEI}} c_{0}}{c_{\mathrm{Li}}} \sqrt{\frac{2 c_{\mathrm{SEI}} t}{D_{\mathrm{sol}} c_{\mathrm{sol}}}} .
$$


Assuming similar solvent concentrations in the SEI and bulk solution, $c_{\mathrm{SEI}} \approx c_{\text {sol }}$, we can use the experimentally observed $\mathrm{Li}$ growth rate to estimate the $\mathrm{Li}^{+}$diffusivity in SEI as $D_{\mathrm{Li}^{+}}^{\mathrm{SEI}} \sim 2 \times 10^{-12}$ $\mathrm{cm}^{2} / \mathrm{s}$, which to our knowledge has not previously been measured. This is a reasonable value, much larger than the solvent diffusivity in SEI (on carbon), $D_{\text {sol }}^{\text {SEI }}$, as well as the solvent selfdiffusivity, ${ }^{56} D_{\text {sol }}=7 \times 10^{-14} \mathrm{~cm}^{2} / \mathrm{s}$, and much smaller than the apparent salt diffusivity in the liquid electrolyte, ${ }^{51} D_{\text {app }}$. Although these estimates establish the relative magnitudes of different transport processes, we note that the standard diffusion equation may not apply at very small $s(t)$, and electric field driven transport like Cabrera-Mott kinetics ${ }^{57}$ may predict a much more rapid (inverse logarithmic) growth of $s(t)$ at the earliest stage of growth, beyond the resolution of the experiments.

After the initial spherical surface growth, the lithium metal protruded out from the electrode and rapidly formed a whisker at $t \sim 30 \mathrm{~s}$. During this stage of growth, a fragment of the electrode (marked by the open arrowhead) was rapidly pushed away from the electrode. This indicates that the growth point of the whisker was at the root, possibly subsurface. ${ }^{58}$ The limiting whisker growth rate corresponds to a mean current density, $I_{2}=42 \mathrm{~mA} / \mathrm{cm}^{2}$, over the root area, if it were by electrochemical deposition. This observed value is much larger than the SEI-limited current, $I_{1}$, and macroscopic exchange current, $I_{0}$, which indicates that bursts of root growth must be temporarily uninhibited by SEI. This may also indicate that such bursts may rely not only on absorbing $\mathrm{Li}^{+}$from the outside, but also on the redistribution of existing Li-atoms beneath the tip, e.g. by relaxation of residual compressive stress by solid-state Nabarro-Herring or Coble creep.

Consistent with this interpretation, the fast root growth was observed to be intermittent. After the whisker became $\sim 1.0 \mu \mathrm{m}$ in length at $t \sim 45 \mathrm{~s}$, it ceased to grow further, and then suddenly at $t \sim 60 \mathrm{~s}$ it grew at approximately the same rate $\left(I_{2}=42 \mathrm{~mA} / \mathrm{cm}^{2}\right)$ in a different direction, forming a kink. Our observations of intermittent root growth with kink formation agrees with reported SEM images of the root-grown lithium whiskers. ${ }^{13,38}$ It is worth noting that the kink may also be a new growth point, ${ }^{13}$ although we did not observe any clear sign of protrusions growing at the kink, possibly because our in-situ TEM experiment was focused at the limited viewing area capturing the initial state of the whisker nucleation/growth. Longer deposition time and viewing at lower magnification may allow us to capture the kink growth mechanism.

In our ETEM (see e.g. Supplementary Movie S3), we can clearly see a gradual darkening of the liquid electrolyte around a newly protruded lithium dendrite, of initial depth scale $\sim 10^{2} \mathrm{~nm}$ (Fig. 4a), which eventually sharpens to a $\sim 10^{1} \mathrm{~nm}$ or even sub-10nm dark skin layer (Fig. 4a) surrounding the low density lithium-metal whisker, as the bright-field TEM image contrast originates mostly from mass-thickness. The density of lithium is $0.534 \mathrm{~g} / \mathrm{cm}^{3}$ which is about one half of DMSO electrolyte $\left(1.10 \mathrm{~g} / \mathrm{cm}^{3}\right)$. We interpret this as liquid electrolyte decomposition by accepting electrons from the electrode, and subsequent formation of SEI constituents that 
contains $\mathrm{LiF}\left(2.64 \mathrm{~g} / \mathrm{cm}^{3}\right), \mathrm{Li}_{2} \mathrm{O}\left(2.01 \mathrm{~g} / \mathrm{cm}^{3}\right)$, etc. with higher salt concentration and average density than the Li metal and the original electrolyte solution. The contrast and the resolution of the ETEM images are also affected by the thickness of the liquid layer in the cell. A thick layer of electrolyte reduces the contrast and the image resolution. In Figure 4, contrast/resolution was better than those in Fig. 2 because electrolyte was wetting only the surface of the electrode (Supplementary Information Figure. S5). In Fig. 2 (see also Supporting Movies S1 and S2), the image resolution was partially improved when the gas pushed the electrolyte away from the view.

From the ETEM observations, we believe the initial SEI at stage I may be critical for the root protrusion mode above, because a stress-induced breakage of SEI passivation layer at stage I-toII transition may give a hole (like fumarole in volcano) that templates future extrusion, which explains the nearly constant width of the dendrite in stage II-IV, as the dendrite has to protrude out of the same hole on the SEI layer.

After the voltage was switched to dissolve the lithium metal whiskers (lithium stripping), the shrinkage took place mostly at the newer segment formed at later stages as shown in Fig. 4(c) (see also Supplementary Movie S4). This can be explained by the variation of the SEI thickness on the whisker. At the voltage range we are considering, liquid electrolyte decomposition and formation of SEI is unavoidable on any surface that conducts electrons. ${ }^{8}$ Since the newly formed segment $\Lambda 2$ had thinner SEI than $\Lambda 1$, lithium ion diffusion out to the electrolyte happens faster at $\Lambda 2$ and dissolution preferentially took place at $\Lambda 2$. The consequence is that the lithium segment near the root hollowed out, leaving an empty SEI shell tube that electrically disconnect the tip part from the base electrode. This created an electrically inactive lithium metal volume ("dead lithium") above the hollow SEI shell tube that could not be dissolved further. This isolated lithium was eventually swept away into the electrolyte, since the hollowed-out SEI tube stems are apparently quite brittle. The reason for the liquid electrolyte convective flow at small scale could be heating-induced convection, electroosmotic / electrocapillary forces, dielectrophoretic electric field induced membrane bending, etc. We believe such micro-flows are ubiquitous and unavoidable in real batteries, and so the nano-lithium/SEI segments that get broken and entrained into the electrolyte then become nano-lithium flotsam in real batteries. Macroscopically, when we open up a secondary battery with lithium metal anode after cycling, we see the originally white separator wet by liquid electrolyte becomes darker colored and has a "dirty" appearance. We think this is because the pores of the separator with liquid electrolyte are filled with these nano-lithium flotsams, which have huge surface area. In an accident with breached packaging (level C engineering goal for Li-metal anode mentioned earlier), when these nano-lithium flotsams are exposed to oxygen, we believe it increases the fire risks. The nano-lithium flotsam may also drift to the counter electrode by Brownian motion and cause spontaneous discharge and high temperature spots.

In this work we have used a gold electrode for lithium deposition due to the ease of fabrication. However, lithium should react with gold to form $\mathrm{Au}-\mathrm{Li}$ intermetallics at an equilibrium voltage of $\sim 0.4 \mathrm{~V} \mathrm{vs} \mathrm{Li}^{+} / \mathrm{Li}^{59}$ Indeed in our experiments, lithiation of the gold electrode can be clearly 
observed when $-3.5 \mathrm{~V}$ vs. LCO was applied, as shown in Fig. 5a (see also Supplementary Movie S5). This data point of ours is roughly consistent with the literature because the typical plateau charging potential of $\mathrm{LCO}$ cathode is $\sim 3.9 \mathrm{~V} \mathrm{vs} \mathrm{Li}^{+} / \mathrm{Li}$, so 3.9-3.5 $\approx 0.4 \mathrm{~V}$. In our in-situ liquid cell experiments, the areas with lighter contrast in Fig. 5 are the lithiated Au-Li nanodomains. Such Au-Li lithiation nanodomains propagated across the electrode inhomogeneously similar to that reported by Zeng et al. ${ }^{60}$

In stark contrast, we did not observe lithiation of the gold electrode itself or Au-Li nanodomains when lithium metal was deposited through the root growth mode as shown in Fig. 2 and 4, where large overpotentials ( $-4.5 \mathrm{~V}$ and $-6.0 \mathrm{~V}$ vs. LCO) were applied. Strangely, the gold electrode appeared inert. Such large overpotentials may promote the formation of denser, thicker SEI on the Au surface, which reduces the rate of Au-Li formation despite a larger thermodynamic driving force of forming Au-Li compared to Fig. 5a case, which could be a kinetic passivation effect like the anodic-protection passivity regime in metal corrosion. The denser and thicker initial SEI is also inductive to causing stress build-up, as the SEI patches tend to connect up and cover the electron-donating surfaces completely, promoting the root growth mode (see Fig. 6). Once the initial SEI are punctured due to stress at stage I-to-II transition, and a hole formed like the "fumarole" of a volcano, the kinetics seems to favor direct lithium metal deposition at the root, instead of Au-Li formation. Although Au-Li can be formed at any time afterwards by chemical reaction according to thermodynamics, we do not actually see Au-Li formation in ETEM, but relatively faster Li deposition rate at the fumarole instead.

The above phenomena may be attributable to a liquid | SEI1 | SEI0 | Au interfacial structure, similar to the bilayer SEI model for carbon electrodes, ${ }^{37,61-63}$ where SEI1 is conductive to $\mathrm{Li}^{+}$but not electrons (like the conventional SEI), and SEI0 is conductive to electrons but not $\mathrm{Li}^{+}$ion or $\mathrm{Li}$ atom (unconventional SEI, playing a role similar to the anodic-protection passivation layer in metal corrosion). If we accept this hypothetical two-layer SEI structure, the nucleation and growth of Lithium metal should occur at SEI1 | SEI0 interface (the "root"). According to this picture, $\mathrm{Au}-\mathrm{Li}$ formation is kinetically throttled by SEI0 because SEI0 does not conduct $\mathrm{Li}^{+}$ion or Li atom, and SEI0 forms only at high overpotentials (-4.5 V and -6.0 V vs. LCO).

The lithium growth mode was distinctly different when an intermediate potential (-4.0 V vs. LCO) was applied. Figure 5b shows the deposition process (see also Supplementary Movie S6). At this potential, growth occurs fairly uniformly over the surfaces, resulting in dense clusters of random surface growth similar to the Eden model ${ }^{64}$ only smoother due to surface tension and surface diffusion. The bud-like protrusions initially resemble the spherical nucleus of Fig. 4 in Stage I, but rather than erupting into narrow whiskers (Stages II-IV), these structures keep growing in all directions and until they become crowded on the surface like cauliflower, and then have to grow outward. At such an intermediate overpotential, the decomposition rate of the liquid electrolyte can be decreased and becomes slower than the lithium deposition rate, and $\mathrm{Li}$ metal grows from the tip instead of the root. Nonetheless, we expect some disconnected SEI molecular fragments mixed in with the growing lithium metal. Finite rates for both reactions 
could be added to the Li/SEI growth model above, leading to a gradual linear (not square-root) current increase at early times. ${ }^{54}$ This implies that the relationship between the SEI formation and the lithium deposition rates is important for determining the Tip vs. Root growth mode. The two modes actually give very distinct morphologies: the root growth mode gives relatively sparsely separated lithium "hairs" or "whiskers" with constant widths, while the tip growth mode (Fig. 5b) gives dense "buds" that can easily grow in width as well as length, so that they end up densely packed in the lateral direction like cauliflowers. It is important to stress these dense "mossy" structures are not true "dendrites", which are sparse, branched fractal structures caused by electrolyte diffusion limitation ahead of the tips, ${ }^{7}$ as observed in copper electrodeposition or solidification. As noted above, lithium growth cannot be limited by liquidstate electrolyte diffusion at these length and time scales and is instead determined by local interfacial kinetics (including diffusion in the SEI film). Our observation reveals that the fast growing SEI at high over potential confines the lithium causing the stress build-up that leads to the root growth of the lithium whiskers. On the other hand, the SEI growth rate is not high enough at low over potential, and SEI may co-deposit with lithium metal to allow electrons to reach the surface, hence the tip growth.

From the observations and analyses above, we suggest the following root growth mechanism in electrodeposition and subsequent shrinkage of the lithium whiskers in electrostripping as schematically shown in Fig. 6. At potential below $-0.5 \mathrm{~V}$ vs $\mathrm{Li}^{+} / \mathrm{Li}$, lithium starts to deposit at the SEI1 | SEI0 interface, preferentially at a nucleation site where SEI0 is thinner, and forms a spherical bud by tip growth, hindered by SEI1 (Stage I). While such an "inverted" SEI0 layer (stopping Li and not electrons) runs contrary to conventional notions about SEI, it has been postulated for carbon anodes ${ }^{37,61-63}$ and is consistent with the absence of the Au-Li nanodomains in our experiments at large overpotentials ( $-4.5 \mathrm{~V}$ and $-6.0 \mathrm{~V}$ vs. LCO). We therefore propose that SEI0 would form in DMSO/LiTFSI when the electrode potential drops below $-0.5 \mathrm{~V}$ vs $\mathrm{Li}^{+} / \mathrm{Li}$. Some SEI0 may also form on the fresh surface and contribute to reducing the growth rate and hardening of the SEI1 film. The net result is that compressive stress arises underneath. When the stress reaches a threshold, SEI1 covering can no longer sustain the stress, especially along the sides near the root. The punctured hole on the initially flat SEI1 acts as the "fumarole" of the volcano, and templates further growth. Lithium then spontaneously breaks out and pushes the top to form a whisker (Stage II), vaguely analogous to the seismic triggering of volcano activities. During this burst of growth, the root may slightly separate from the original SEI0 base, so that fresh lithium can freely diffuse and deposit under the root, causing the whisker to extend. Solvent molecules are too large and slow to diffuse into the root to hinder electrodeposition, but as lithium fills in the relaxed space beneath, the whisker growth stops (Stage III). The process then repeats itself, as further stress increase leads to another SEI1 film rupture and root growth of the next whisker segment (Stage IV).

Besides the applied potential, various factors can affect the relative rates of lithium electrodeposition and SEI decomposition, and the composition of the SEI, such as the operating 
temperature, electrolyte compositions, electrode materials, and the surface roughness. Extensive parametric studies will be required to understand the complex interactions of these factors and quantitatively predict the lithium deposition mechanism (Tip vs. Root) and rate. This is a complex nonlinear problem in chemo-mechanics leading to intermittent growth, similar to stickslip motion in friction. A subsurface compressive stress is known to be a driving force to form Sn whiskers on a substrate. ${ }^{65-67}$ The dominant direction of the residual stress accumulated subsurface may change from time period to time period due to changing boundary conditions, inducing stop-and-go, time-dependent "thrust" directions and formation of kinks. Because the bulk melting point $T_{\mathrm{M}}$ of lithium metal is $454 \mathrm{~K}$, at room temperature $T / T_{\mathrm{M}}=0.66$, surface and/or lattice diffusion of lithium atoms is expected to be facile. Therefore the root growth of lithium hair driven by subsurface compressive stress may be well described by linear-response Coble/Nabarro-Herring diffusional creep theory ${ }^{68,69}$ It was also reported for lithium that a subsurface structure may be responsible for dendrite nucleation ${ }^{58}$.

Because of the time lag between the formations of the whisker segments, the thickness of the SEI formed on the whisker can vary, and the dissolution is expected to be faster where the SEI is thinner. This causes regions near the electrode to be the primary sites of dissolution in lithium stripping, leaving the lithium toward the tip isolated. These dead volumes may be fully disconnected from the electrode and become nano-lithium flotsams or partially maintain mechanical connection with a SEI shell tube. In either case, there will be no electric path to the isolated lithium.

Next-generation energy storage technologies such as Li-S $\mathrm{S}^{70,71}$ and Li-air(oxygen) ${ }^{72,73}$ batteries can greatly benefit from the use of Li metal anode. In-situ ETEM observations shed light on detailed growth, shrinkage, electrical isolation and mechanical spallation (flotsam) mechanisms

of the lithium whiskers. We have also directly visualized liquid electrolyte decomposition and SEI formation. The SEI formation is a nanoscale phenomenon (irreversible side reaction) that competes with lithium metal deposition in consuming the Faradaic current, and also directly impacts the root vs. tip growth mechanism of the lithium dendrite. Electrolyte additives could modify the structure and properties of the naturally formed SEI, and thus influence the lithium dendrite instability. Since the naturally formed SEI is very thin $\left(\sim 10^{1} \mathrm{~nm}\right.$ to sub-10 nm), this would also mean that an artificial SEI surface decoration (like BN or oxide treatment) of the electrode could significantly improve the surface instability. These understanding may help to develop protrusion suppression methods over a wide range of overpotentials.

\section{Acknowledgements}

We acknowledge Honda R\&D Co., Ltd. for financial support and fabrication/supply of LEEC. MZB acknowledges support from the Global Climate and Energy Project at Stanford University and the US Department of Energy, Basic Energy Sciences through the SUNCAT Center for Interface Science and Catalysis. This work made use of the MRSEC Shared Experimental 
Facilities at MIT, supported by the National Science Foundation under award number DMR1419807 and ECCS-1610806.

\section{References}

(1) Aurbach, D.; Cohen, Y. The Application of Atomic Force Microscopy for the Study of Li Deposition Processes. J. Electrochem. Soc. 1996, 143, 3525-3532.

(2) Aurbach, D.; Zinigrad, E.; Cohen, Y.; Teller, H. A Short Review of Failure Mechanisms of Lithium Metal and Lithiated Graphite Anodes in Liquid Electrolyte Solutions. Solid State Ion. 2002, 148, 405-416.

(3) Whittingham, M. S. History, Evolution, and Future Status of Energy Storage. Proc. IEEE 2012, 100, 1518-1534.

(4) Xu, W.; Wang, J.; Ding, F.; Chen, X.; Nasybulin, E.; Zhang, Y.; Zhang, J.-G. Lithium Metal Anodes for Rechargeable Batteries. Energy Environ. Sci. 2014, 7, 513-537.

(5) Whittingham, M. S. Electrical Energy Storage and Intercalation Chemistry. Science 1976, 192, 1126-1127.

(6) Li, Z.; Huang, J.; Yann Liaw, B.; Metzler, V.; Zhang, J. A Review of Lithium Deposition in Lithium-Ion and Lithium Metal Secondary Batteries. J. Power Sources 2014, 254, 168-182.

(7) Bai, P.; Li, J.; Brushett, F. R.; Bazant, M. Z. Transition of Lithium Growth Mechanisms in Liquid Electrolytes. Energy Environ. Sci. 2016, 9, 3221-3229.

(8) Goodenough, J. B.; Kim, Y. Challenges for Rechargeable Li Batteries. Chem. Mater. 2010, 22, 587-603.

(9) Chianelli, R. R. Microscopic Studies of Transition Metal Chalcogenides. J. Cryst. Growth 1976, 34, 239-244.

(10) Epelboin, I.; Froment, M.; Garreau, M.; Thevenin, J.; Warin, D. Behavior of Secondary Lithium and Aluminum - Lithium Electrodes in Propylene Carbonate. J. Electrochem. Soc. 1980, 127, 2100-2104.

(11) Gireaud, L.; Grugeon, S.; Laruelle, S.; Yrieix, B.; Tarascon, J.-M. Lithium Metal Stripping/Plating Mechanisms Studies: A Metallurgical Approach. Electrochem. Commun. 2006, 8, 1639-1649.

(12) Rosso, M.; Brissot, C.; Teyssot, A.; Dollé, M.; Sannier, L.; Tarascon, J.-M.; Bouchet, R.; Lascaud, S. Dendrite Short-Circuit and Fuse Effect on Li/Polymer/Li Cells. Electrochimica Acta 2006, 51, 5334-5340. 
(13) Steiger, J.; Kramer, D.; Mönig, R. Mechanisms of Dendritic Growth Investigated by in Situ Light Microscopy during Electrodeposition and Dissolution of Lithium. J. Power Sources 2014, 261, 112-119.

(14) Steiger, J.; Kramer, D.; Mönig, R. Microscopic Observations of the Formation, Growth and Shrinkage of Lithium Moss during Electrodeposition and Dissolution. Electrochimica Acta 2014, 136, 529-536.

(15) Crowther, O.; West, A. C. Effect of Electrolyte Composition on Lithium Dendrite Growth. J. Electrochem. Soc. 2008, 155, A806-A811.

(16) Monroe, C.; Newman, J. Dendrite Growth in Lithium/Polymer Systems A Propagation Model for Liquid Electrolytes under Galvanostatic Conditions. J. Electrochem. Soc. 2003, 150, A1377-A1384.

(17) Barton, J. L.; Bockris, J. O. The Electrolytic Growth of Dendrites from Ionic Solutions. Proc. R. Soc. Lond. Math. Phys. Eng. Sci. 1962, 268, 485-505.

(18) Diggle, J. W.; Despic, A. R.; Bockris, J. O. The Mechanism of the Dendritic Electrocrystallization of Zinc. J. Electrochem. Soc. 1969, 116, 1503-1514.

(19) Yamaki, J.; Tobishima, S.; Hayashi, K.; Saito, K.; Nemoto, Y.; Arakawa, M. A Consideration of the Morphology of Electrochemically Deposited Lithium in an Organic Electrolyte. J. Power Sources 1998, 74, 219-227.

(20) Ota, H.; Shima, K.; Ue, M.; Yamaki, J. Effect of Vinylene Carbonate as Additive to Electrolyte for Lithium Metal Anode. Electrochimica Acta 2004, 49, 565-572.

(21) Naoi, K.; Mori, M.; Naruoka, Y.; Lamanna, W. M.; Atanasoski, R. The Surface Film Formed on a Lithium Metal Electrode in a New Imide Electrolyte, Lithium Bis(perfluoroethylsulfonylimide) [ LiN ( C 2 F 5 SO 2 ) 2 ]. J. Electrochem. Soc. 1999, 146, 462-469.

(22) Mogi, R.; Inaba, M.; Jeong, S.-K.; Iriyama, Y.; Abe, T.; Ogumi, Z. Effects of Some Organic Additives on Lithium Deposition in Propylene Carbonate. J. Electrochem. Soc. 2002, 149, A1578-A1583.

(23) Zhamu, A.; Chen, G.; Liu, C.; Neff, D.; Fang, Q.; Yu, Z.; Xiong, W.; Wang, Y.; Wang, X.; Jang, B. Z. Reviving Rechargeable Lithium Metal Batteries: Enabling next-Generation High-Energy and High-Power Cells. Energy Environ. Sci. 2012, 5, 5701-5707.

(24) Zheng, G.; Lee, S. W.; Liang, Z.; Lee, H.-W.; Yan, K.; Yao, H.; Wang, H.; Li, W.; Chu, S.; Cui, Y. Interconnected Hollow Carbon Nanospheres for Stable Lithium Metal Anodes. Nat. Nanotechnol. 2014, 9, 618-623.

(25) Ding, F.; Xu, W.; Graff, G. L.; Zhang, J.; Sushko, M. L.; Chen, X.; Shao, Y.; Engelhard, M. H.; Nie, Z.; Xiao, J.; et al. Dendrite-Free Lithium Deposition via Self-Healing Electrostatic Shield Mechanism. J. Am. Chem. Soc. 2013, 135, 4450-4456.

(26) Osaka, T.; Homma, T.; Momma, T.; Yarimizu, H. In Situ Observation of Lithium Deposition Processes in Solid Polymer and Gel Electrolytes. J. Electroanal. Chem. 1997, 421, 153-156.

(27) Orsini, F.; Du Pasquier, A.; Beaudoin, B.; Tarascon, J. M.; Trentin, M.; Langenhuizen, N.; De Beer, E.; Notten, P. In Situ Scanning Electron Microscopy (SEM) Observation of Interfaces within Plastic Lithium Batteries. J. Power Sources 1998, 76, 19-29.

(28) Orsini, F.; du Pasquier, A.; Beaudouin, B.; Tarascon, J. M.; Trentin, M.; Langenhuizen, N.; de Beer, E.; Notten, P. In Situ SEM Study of the Interfaces in Plastic Lithium Cells. $J$. Power Sources 1999, 81-82, 918-921. 
(29) Porter, D. A.; Easterling, K. E. Phase Transformations in Metals and Alloys; 2nd ed.; Chapman \& Hall: London, 1992.

(30) Brady, R. M.; Ball, R. C. Fractal Growth of Copper Electrodeposits. Nature 1984, 309, 225-229.

(31) Grier, D.; Ben-Jacob, E.; Clarke, R.; Sander, L. M. Morphology and Microstructure in Electrochemical Deposition of Zinc. Phys. Rev. Lett. 1986, 56, 1264-1267.

(32) Trigueros, P. P.; Claret, J.; Mas, F.; Sagués, F. Pattern Morphologies in Zinc Electrodeposition. J. Electroanal. Chem. Interfacial Electrochem. 1991, 312, 219-235.

(33) Fleury, V.; Rosso, M.; Chazalviel, J.-N.; Sapoval, B. Experimental Aspects of Dense Morphology in Copper Electrodeposition. Phys. Rev. A 1991, 44, 6693-6705.

(34) Léger, C.; Elezgaray, J.; Argoul, F. Dynamical Characterization of One-Dimensional Stationary Growth Regimes in Diffusion-Limited Electrodeposition Processes. Phys. Rev. E 1998, 58, 7700-7709.

(35) Brissot, C.; Rosso, M.; Chazalviel, J.-N.; Lascaud, S. Dendritic Growth Mechanisms in Lithium/Polymer Cells. J. Power Sources 1999, 81-82, 925-929.

(36) Rosso, M.; Gobron, T.; Brissot, C.; Chazalviel, J.-N.; Lascaud, S. Onset of Dendritic Growth in Lithium/Polymer Cells. J. Power Sources 2001, 97-98, 804-806.

(37) Tang, M.; Lu, S.; Newman, J. Experimental and Theoretical Investigation of SolidElectrolyte-Interphase Formation Mechanisms on Glassy Carbon. J. Electrochem. Soc. 2012, 159, A1775-A1785.

(38) Dollé, M.; Sannier, L.; Beaudoin, B.; Trentin, M.; Tarascon, J.-M. Live Scanning Electron Microscope Observations of Dendritic Growth in Lithium/Polymer Cells. Electrochem. Solid-State Lett. 2002, 5, A286-A289.

(39) Huang, J. Y.; Zhong, L.; Wang, C. M.; Sullivan, J. P.; Xu, W.; Zhang, L. Q.; Mao, S. X.; Hudak, N. S.; Liu, X. H.; Subramanian, A.; et al. In Situ Observation of the Electrochemical Lithiation of a Single SnO2 Nanowire Electrode. Science 2010, 330, 15151520.

(40) Liu, X. H.; Liu, Y.; Kushima, A.; Zhang, S.; Zhu, T.; Li, J.; Huang, J. Y. In Situ TEM Experiments of Electrochemical Lithiation and Delithiation of Individual Nanostructures. Adv. Energy Mater. 2012, 2, 722-741.

(41) de Jonge, N.; Ross, F. M. Electron Microscopy of Specimens in Liquid. Nat. Nanotechnol. 2011, 6, 695-704.

(42) Liao, H.-G.; Cui, L.; Whitelam, S.; Zheng, H. Real-Time Imaging of Pt3Fe Nanorod Growth in Solution. Science 2012, 336, 1011-1014.

(43) Sun, M.; Liao, H.-G.; Niu, K.; Zheng, H. Structural and Morphological Evolution of Lead Dendrites during Electrochemical Migration. Sci. Rep. 2013, 3.

(44) Sacci, R. L.; Dudney, N. J.; More, K. L.; Parent, L. R.; Arslan, I.; Browning, N. D.; Unocic, R. R. Direct Visualization of Initial SEI Morphology and Growth Kinetics during Lithium Deposition by in Situ Electrochemical Transmission Electron Microscopy. Chem. Commun. 2014, 50, 2104-2107.

(45) Zeng, Z.; Liang, W.-I.; Liao, H.-G.; Xin, H. L.; Chu, Y.-H.; Zheng, H. Visualization of Electrode-Electrolyte Interfaces in LiPF6/EC/DEC Electrolyte for Lithium Ion Batteries via in Situ TEM. Nano Lett. 2014, 14, 1745-1750.

(46) Mehdi, B. L.; Stevens, A.; Qian, J.; Park, C.; Xu, W.; Henderson, W. A.; Zhang, J.-G.; Mueller, K. T.; Browning, N. D. The Impact of Li Grain Size on Coulombic Efficiency in Li Batteries. Sci. Rep. 2016, 6, 34267. 
(47) Kushima, A.; Koido, T.; Fujiwara, Y.; Kuriyama, N.; Kusumi, N.; Li, J.

Charging/Discharging Nanomorphology Asymmetry and Rate-Dependent Capacity Degradation in Li-Oxygen Battery. Nano Lett. 2015.

(48) Sacci, R. L.; Black, J. M.; Balke, N.; Dudney, N. J.; More, K. L.; Unocic, R. R. Nanoscale Imaging of Fundamental Li Battery Chemistry: Solid-Electrolyte Interphase Formation and Preferential Growth of Lithium Metal Nanoclusters. Nano Lett. 2015, 15, 2011-2018.

(49) Marinaro, M.; Balasubramanian, P.; Gucciardi, E.; Theil, S.; Jörissen, L.; WohlfahrtMehrens, M. Importance of Reaction Kinetics and Oxygen Crossover in Aprotic Li-O2 Batteries Based on a Dimethyl Sulfoxide Electrolyte. ChemSusChem 2015, 8, 3139-3145.

(50) Aoki, K. Theory of Ultramicroelectrodes. Electroanalysis 1993, 5, 627-639.

(51) Valøen, L. O.; Reimers, J. N. Transport Properties of LiPF6-Based Li-Ion Battery Electrolytes. J. Electrochem. Soc. 2005, 152, A882-A891.

(52) Munichandraiah, N.; Scanlon, L. G.; Marsh, R. A. Surface Films of Lithium: An Overview of Electrochemical Studies. J. Power Sources 1998, 72, 203-210.

(53) Ploehn, H. J.; Ramadass, P.; White, R. E. Solvent Diffusion Model for Aging of LithiumIon Battery Cells. J. Electrochem. Soc. 2004, 151, A456-A462.

(54) Pinson, M. B.; Bazant, M. Z. Theory of SEI Formation in Rechargeable Batteries: Capacity Fade, Accelerated Aging and Lifetime Prediction. J. Electrochem. Soc. 2013, 160, A243A250.

(55) Gogoana, R.; Pinson, M. B.; Bazant, M. Z.; Sarma, S. E. Internal Resistance Matching for Parallel-Connected Lithium-Ion Cells and Impacts on Battery Pack Cycle Life. J. Power Sources 2014, 252, 8-13.

(56) Ilyina, E.; Daragan, V. Self-Diffusion of Dimethyl Sulfoxide and Dimethylformamide in Solutions and Gels of Cellulose Acetates by Pulsed Field Gradient NMR. Macromolecules 1994, 27, 3759-3763.

(57) Cabrera, N.; Mott, N. F. Theory of the Oxidation of Metals. Rep. Prog. Phys. 1949, 12, 163.

(58) Harry, K. J.; Hallinan, D. T.; Parkinson, D. Y.; MacDowell, A. A.; Balsara, N. P. Detection of Subsurface Structures underneath Dendrites Formed on Cycled Lithium Metal Electrodes. Nat. Mater. 2014, 13, 69-73.

(59) Taillades, G.; Benjelloun, N.; Sarradin, J.; Ribes, M. Metal-Based Very Thin Film Anodes for Lithium Ion Microbatteries. Solid State Ion. 2002, 152-153, 119-124.

(60) Zeng, Z.; Liang, W.-I.; Chu, Y.-H.; Zheng, H. In Situ TEM Study of the Li-Au Reaction in an Electrochemical Liquid Cell. Faraday Discuss. 2015, 176, 95-107.

(61) Peled, E. Lithium Batteries; Academic Press: New York, NY, 1983.

(62) Peled, E.; Golodnitsky, D.; Ardel, G.; Menachem, C.; Bar Tow, D.; Eshkenazy, V. M19. Mater. Res. Soc. Symp. Proc. 1995, 393, 209.

(63) Peled, E.; Golodnitsky, D.; Ardel, G. Advanced Model for Solid Electrolyte Interphase Electrodes in Liquid and Polymer Electrolytes. J. Electrochem. Soc. 1997, 144, L208-L210.

(64) Barbasi, A.-L.; Stanley, H. E. Fractal Concepts in Surface Growth; Cambridge University Press, 1995.

(65) Franks, J. Growth of Whiskers in the Solid Phase. Acta Metall. 1958, 6, 103-109.

(66) Tu, K. N. Irreversible Processes of Spontaneous Whisker Growth in Bimetallic Cu-Sn ThinFilm Reactions. Phys. Rev. B 1994, 49, 2030-2034.

(67) Sobiech, M.; Wohlschlögel, M.; Welzel, U.; Mittemeijer, E. J.; Hügel, W.; Seekamp, A.; Liu, W.; Ice, G. E. Local, Submicron, Strain Gradients as the Cause of Sn Whisker Growth. Appl. Phys. Lett. 2009, 94, 221901. 
(68) Tian, L.; Li, J.; Sun, J.; Ma, E.; Shan, Z.-W. Visualizing Size-Dependent Deformation Mechanism Transition in Sn. Sci. Rep. 2013, 3.

(69) Sun, J.; He, L.; Lo, Y.-C.; Xu, T.; Bi, H.; Sun, L.; Zhang, Z.; Mao, S. X.; Li, J. Liquid-like Pseudoelasticity of Sub-10-Nm Crystalline Silver Particles. Nat. Mater. 2014, 13, 10071012.

(70) Ji, X.; Lee, K. T.; Nazar, L. F. A Highly Ordered Nanostructured Carbon-sulphur Cathode for Lithium-sulphur Batteries. Nat. Mater. 2009, 8, 500-506.

(71) Bruce, P. G.; Freunberger, S. A.; Hardwick, L. J.; Tarascon, J.-M. Li-O2 and Li-S Batteries with High Energy Storage. Nat. Mater. 2012, 11, 19-29.

(72) Girishkumar, G.; McCloskey, B.; Luntz, A. C.; Swanson, S.; Wilcke, W. Lithium-Air Battery: Promise and Challenges. J. Phys. Chem. Lett. 2010, 1, 2193-2203.

(73) Zhu, Z.; Kushima, A.; Yin, Z.; Qi, L.; Amine, K.; Lu, J.; Li, J. Anion-Redox Nanolithia Cathodes for Li-Ion Batteries. Nat. Energy Accepted, DOI: 10.1038/NENERGY.2016.111. 


\section{Figures:}
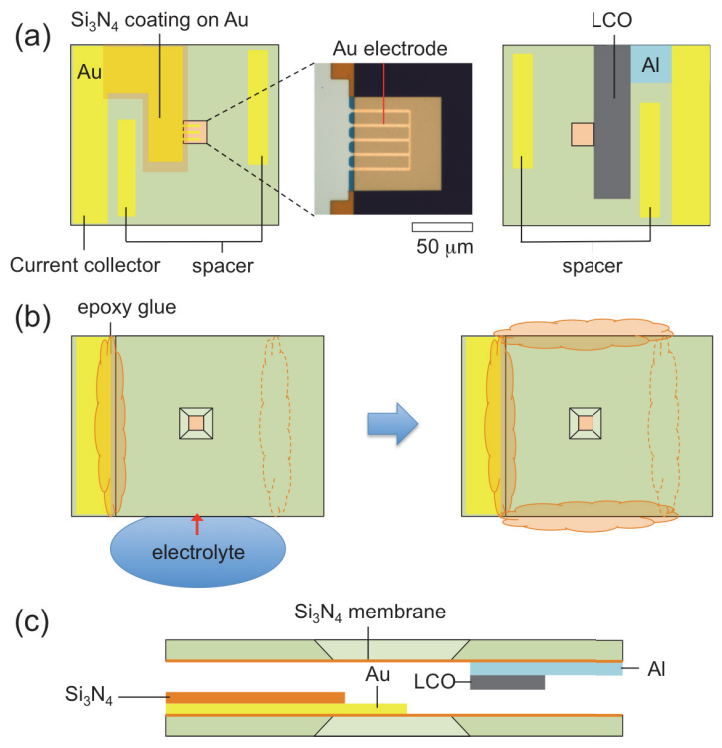

Figure 1. (a) Schematic illustration of the liquid confining cell. The cell consists of two chips. The one with $\mathrm{Au}$ electrode patterned on the silicon nitride membrane has Au current collector coated with $\mathrm{Si}_{3} \mathrm{~N}_{4}$ insulating layer to prevent the electrolyte exposure (left). The optical micrograph image is the magnified view of the $\mathrm{Au}$ electrode. $\mathrm{LiCoO}_{2}(\mathrm{LCO})$ is sputtered on the $\mathrm{Al}$ film connected to the Au current collector as Li source (right). Both chips have Au spacers to secure a space for the electrolyte. (b) Procedure of the chip assembly. (c) Cross section view of the assembled cell near the silicon nitride membrane windows.

(a)

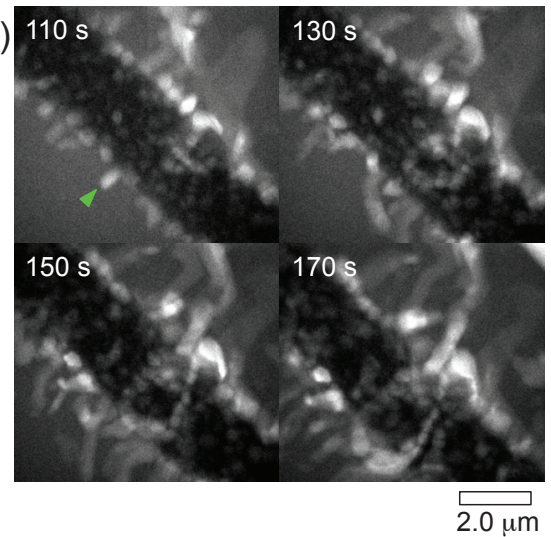

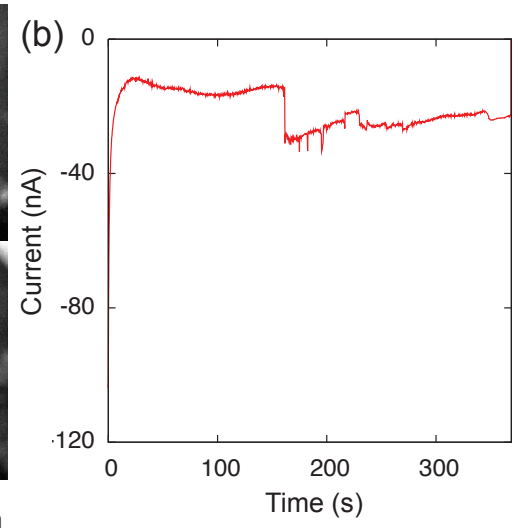

(c)

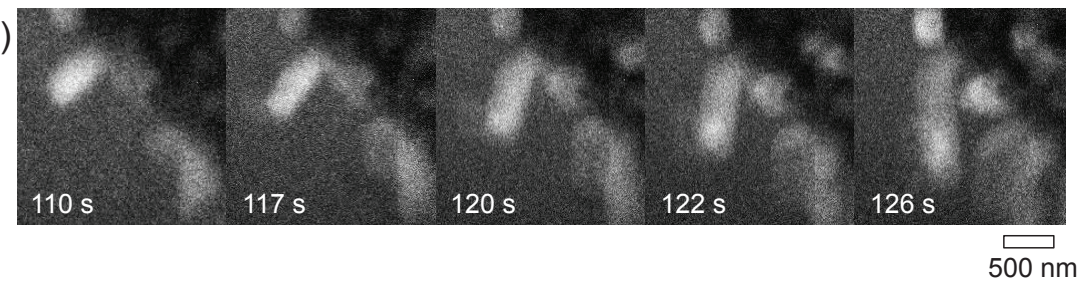

Figure 2. (a) Sequential TEM images of the Li whisker growth at $-4.5 \mathrm{~V}$ vs. LCO (Supplementary Movie S1). (b) Change in the electric current during the lithium deposition. (c) Close up view of a single Li hair growth process indicated by the arrowhead in (a). 

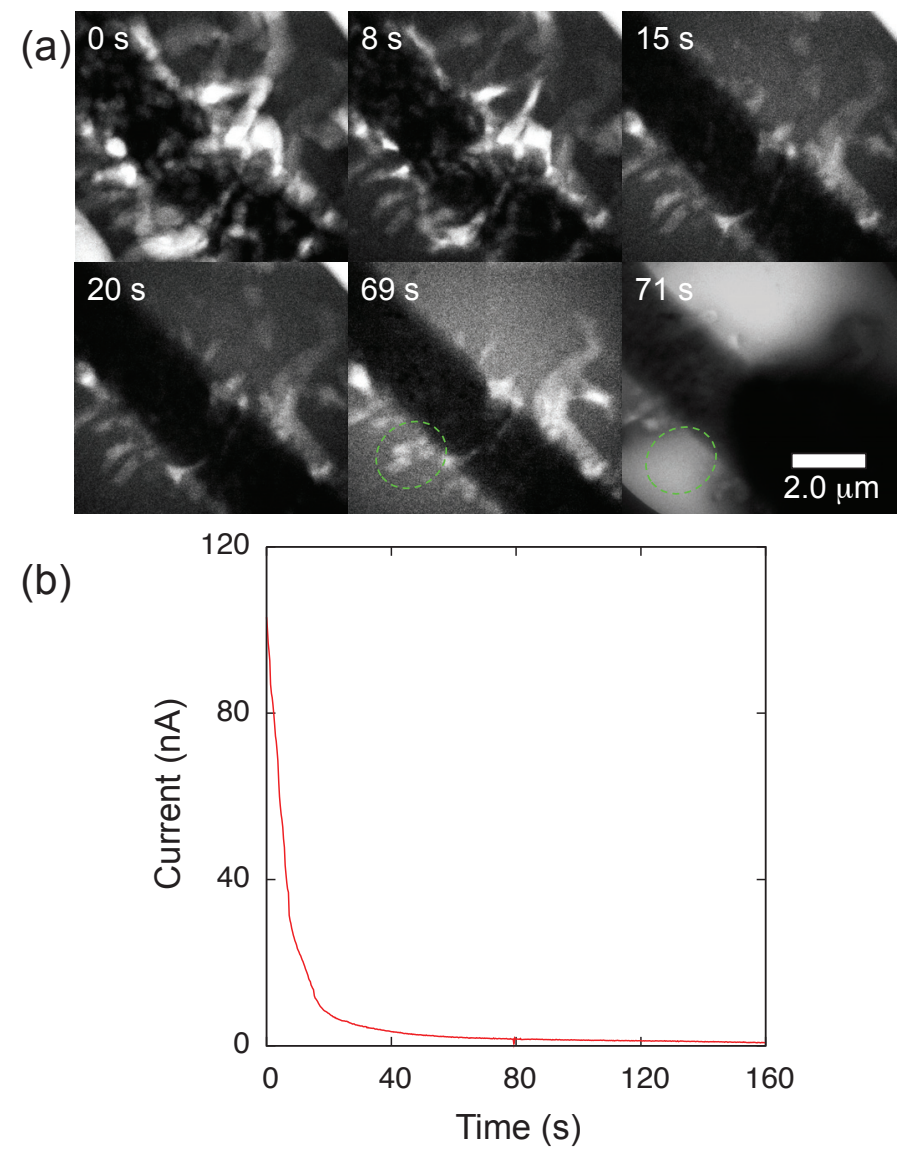

Figure 3. (a) Decomposition process of the Li whisker at $1.0 \mathrm{~V}$ vs. LCO biasing (Supplementary Movie S2). The lithium hair decomposed rapidly in the first 20 seconds and the rate became significantly slower at $t>20 \mathrm{~s}$. A piece of Li marked in the dashed line was swept away into the electrolyte. (b) Change in the electrical current during the $\mathrm{Li}$ decomposition. The fast decay of the current corresponds to the $\mathrm{Li}$ decomposition at $t<20 \mathrm{~s}$. 
(a)

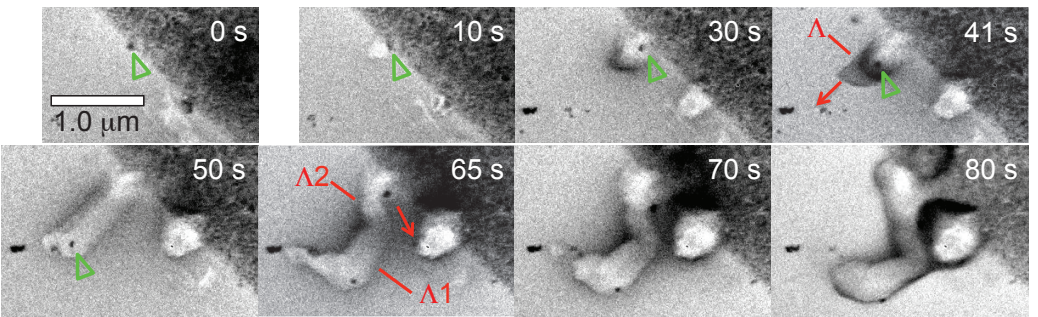

(b)

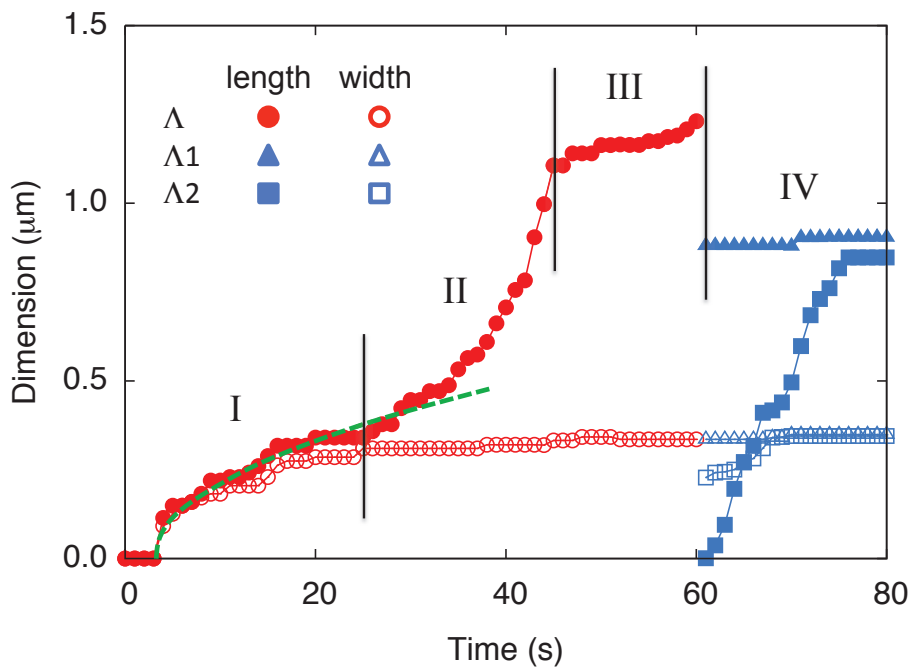

(c)



Figure 4. (a) A temporal evolution of a single Li whisker growth under $-6.0 \mathrm{~V}$ vs. LCO biasing (Supplementary Movie S3). (b) Change in the length and the width of the lithium whisker $\Lambda(\Lambda 1$ and $\Lambda 2$ after the kink formation) as indicated in (a). Dashed line is the square root fitting of the data plot at the initial stage of the whisker formation. (c) The decomposition process at $0.0 \mathrm{~V}$ vs. LCO (Supplementary Movie S4). 


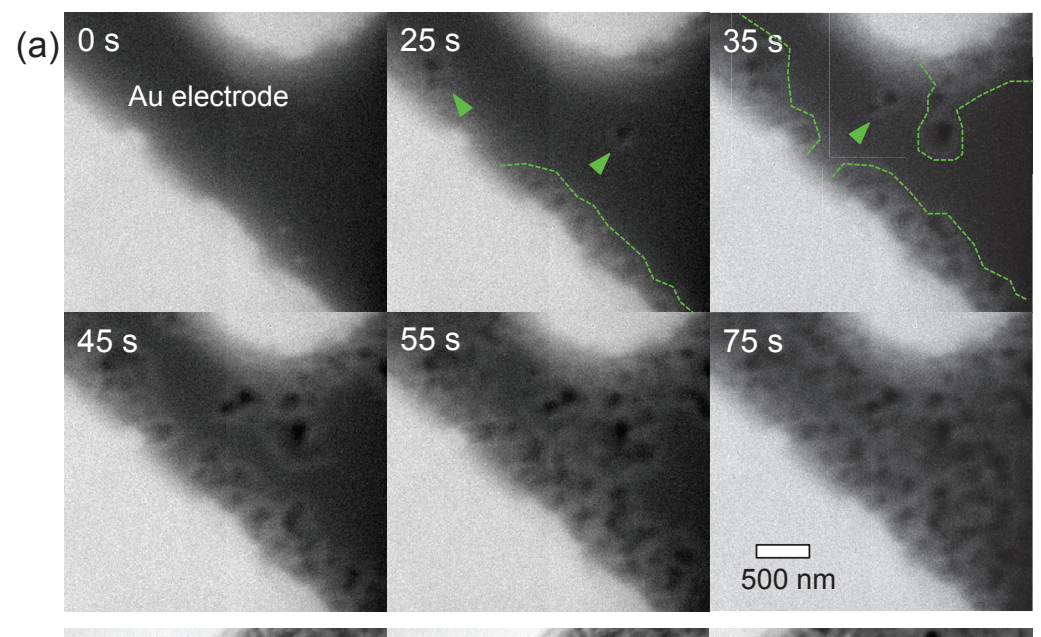

(b) $0 \mathrm{~s}$

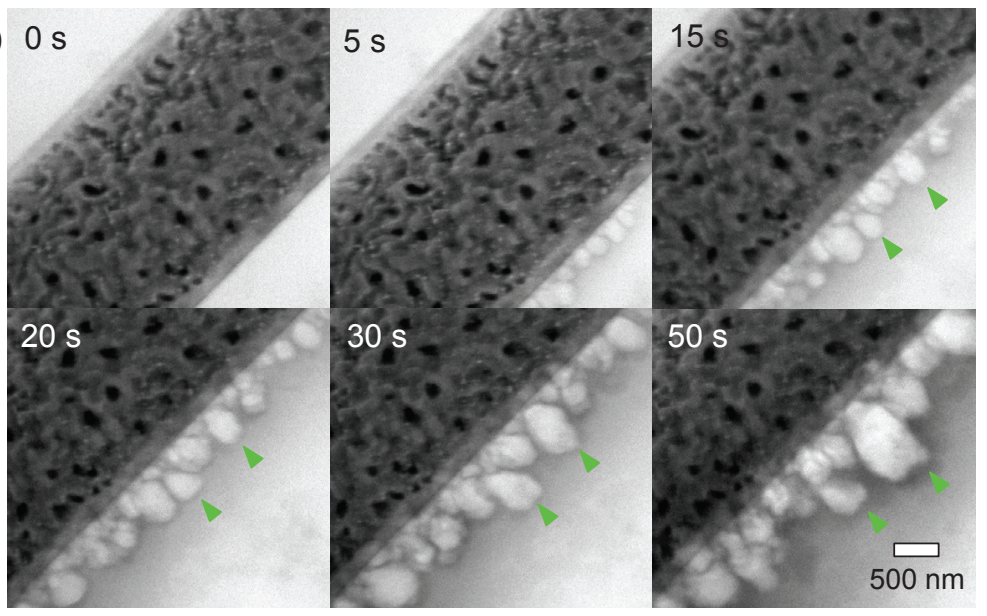

Figure 5. (a) Lithiation of the gold electrode at -3.5 V vs. LCO (Supplementary Movie S5). The lithiation was randomly initiated as indicated by the arrowheads and propagated inhomogeneously across the electrode (the boundaries are marked by the dashed lines). (b) Mossy lithium grown on the gold electrode at $-4.0 \mathrm{~V}$ vs. LCO (Supplementary Movie S6). The morphology of the tips changed as in the growth process (marked by the arrowheads), indicating the deposition took place at the tip. 
(a) Small overpotential "mossy growth"

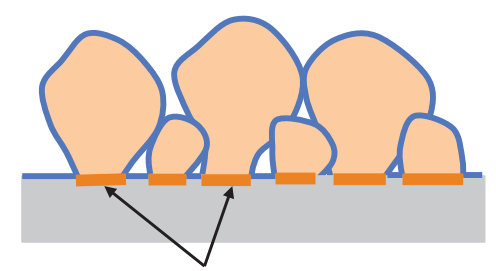

Nucleation sites (b) Large overpotential "whisker growth"

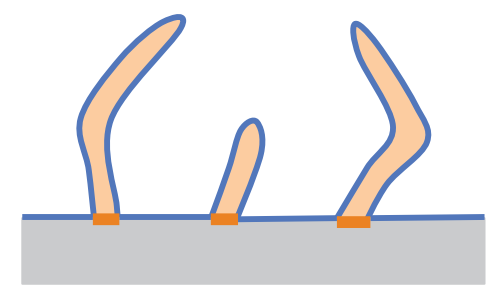

(c) $t<0$

$t=0 \quad$ Nucleation


Stage 1: Hindered surface growth

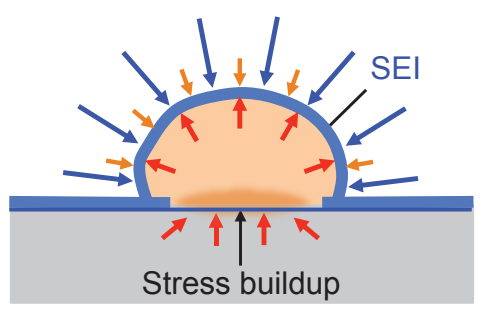

Stage 2: Fast root growth

Stage 3: Hindered root growth
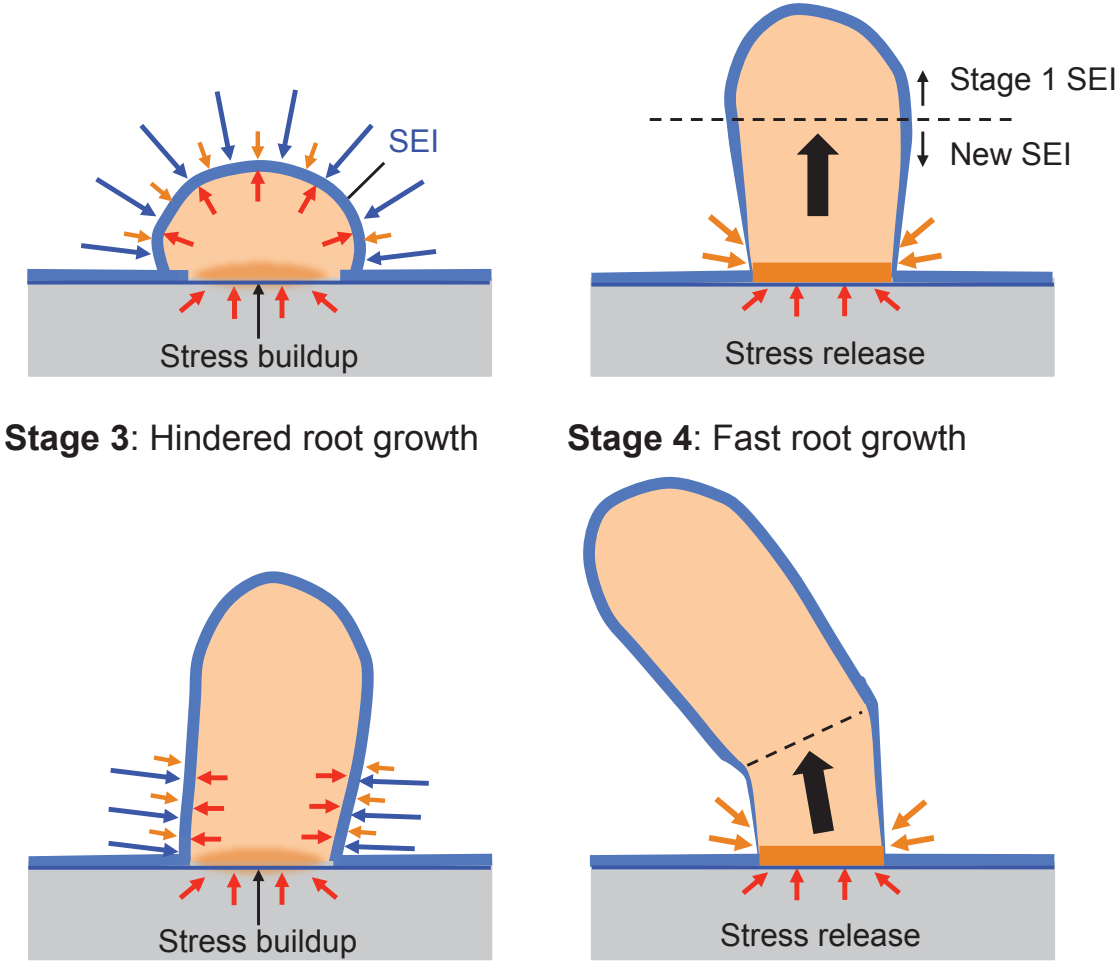

Stage 4: Fast root growth

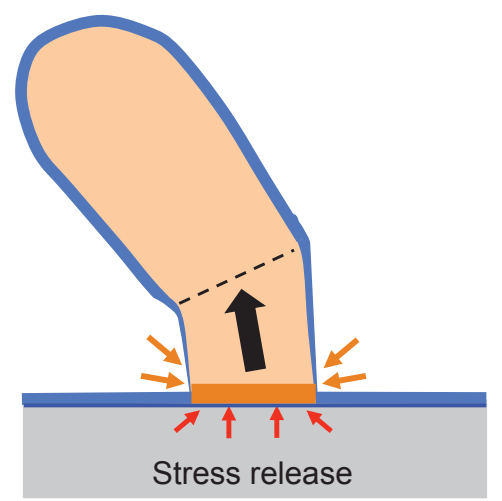

Figure 6. Schematic illustration explaining root growth mechanism of lithium whiskers. 
Movies: http://li.mit.edu/S/lh/Movie/

Movie S1. Li whiskers grown at $-4.5 \mathrm{~V}$ vs. LCO (played at 10x original speed).

Movie S2. Li whiskers dissolved at $1.0 \mathrm{~V}$ vs. LCO (played at 10x original speed).

Movie S3. A single whisker grown at $-6.0 \mathrm{~V}$ vs. LCO (played at 10x original speed).

Movie S4. A single whisker dissolved at 0.0 V vs. LCO (played at 10x original speed).

Movie S5. Lithiation of the Au electrode at $-3.5 \mathrm{~V}$ vs. LCO (played at 10x original speed).

Movie S6. Mossy lithium grown at $-4.0 \mathrm{~V}$ vs. LCO (played at 10x original speed).

\section{Graphical abstract}



